\title{
Large Extra Dimensions and Cosmological Problems
}

\author{
Glenn D. Starkman ${ }^{1}$, Dejan Stojkovic ${ }^{1}$ and Mark Trodden ${ }^{2}$ \\ ${ }^{1}$ Department of Physics \\ Case Western Reserve University \\ Cleveland, OH 44106-7079, USA \\ ${ }^{2}$ Department of Physics \\ Syracuse University \\ Syracuse, NY, 13244-1130 USA
}

\begin{abstract}
We consider a variant of the brane-world model in which the universe is the direct product of a Friedmann, Robertson-Walker (FRW) space and a compact hyperbolic manifold of dimension $d \geq 2$. Cosmology in this space is particularly interesting. The dynamical evolution of the space-time leads to the injection of a large entropy into the observable (FRW) universe. The exponential dependence of surface area on distance in hyperbolic geometry makes this initial entropy very large, even if the CHM has relatively small diameter (in fundamental units). This provides an attractive reformulation of the cosmological entropy problem, in which the large entropy is a consequence of the topology, though we would argue that a final solution of the entropy problem requires a dynamical explanation of the topology of spacetime. Nevertheless, it is reassuring that this entropy can be achieved within the holographic limit if the ordinary FRW space is also a compact hyperbolic manifold. In addition, the very large statistical averaging inherent in the collapse of the initial entropy onto the brane acts to smooth out initial inhomogeneities. This smoothing is then sufficient to account for the current homogeneity of the universe. With only mild fine-tuning, the current flatness of the universe can also then be understood. Finally, recent brane-world approaches to the hierarchy problem can be readily realized within this framework.
\end{abstract}

CWRU-P12-00

SU-GP-00/12-1

hep-th/??????? 


\section{Introduction}

The standard initial value problems of Big Bang cosmology are usually addressed by a period of cosmic inflation [1], to which viable alternatives have been elusive. (See however [2, [3].) In this paper, we will consider the status of these problems in the context of the idea that our $3+1$ dimensional universe is only a submanifold (3-brane) on which Standard Model fields are confined inside a higher dimensional space [4, 5, 6, 07. In such models, only gravitons and other geometric degrees of freedom may propagate in the bulk space-time. An important motivation for these theories has been to solve the so-called hierarchy problem - explaining the largeness of the Planck mass compared to the scales characterizing other interactions, in particular to the weak scale. If $M_{F}$ is the actual fundamental scale of gravitational interactions, and if the volume of the extra dimensional d-manifold is $V_{\text {extra }}$, then [4, 5, 6, 0] by Gauss's law, at distances larger than the inverse mass of the lightest Kaluza Klein (KK) mode in the theory, the gravitational force will follow an inverse square law with an effective coupling of

$$
M_{\mathrm{pl}}^{-2}=M_{F}^{-(d+2)} V_{\text {extra }}^{-1} .
$$

The canonical realization of this scenario [5] assumed that the extra-dimensional manifold, $\mathcal{M}_{\text {extra }}$, was a $d$-torus of large spatial extent (in fundamental units, $\left.M_{F}^{-1}\right)$. In that case, $M_{F} \gtrsim 50 \mathrm{TeV}$ is consistent with existing particle physics and cosmological phenomenology for $d \geq 2$. However, from many points of view $d$-tori are special. Because they admit flat (Euclidean) geometries, they have no intrinsic geometric scale, and so there is no a priori reason that they should have such a large extent. There is also no gap in the graviton spectrum to the first KK mode. Further, compact manifolds which admit a flat geometry are a set of measure zero in the space of $d$-manifolds.

Recently, it was argued [8] that if the extra dimensions comprised a compact hyperbolic manifold (CHM) then the same volume suppression of gravity could be obtained, and all constraints avoided with a manifold whose radius is only $\mathcal{O}(30) M_{F}^{-1}$. In this case $M_{F} \gtrsim 1 \mathrm{TeV}$ is allowed and the observed gauge hierarchy is a consequence of the topology of space. For $d=2$ and 3 , most manifolds are compact hyperbolic. (For $d>3$, there is no complete classification of compact manifolds; indeed, most compact manifolds probably admit no homogeneous geometry.) These manifolds can be obtained from their better known universal covering space $H^{d}$ by "modding-out" by 
a (freely-acting) discrete subgroup $\Gamma$ of the isometry group of $H^{d}$. (Just as $d$-tori are obtained by modding out d-dimensional Euclidean space $E^{d}$ by a freely-acting discrete subgroup of the Galilean group in $d$-dimensions.) If the structure of the full manifold is

$$
\Sigma_{d+4}=\mathbf{R} \times \mathcal{M}_{F R W} \times \mathcal{M}_{\text {extra }}
$$

then the metric on such a space can be written as

$$
d s^{2}=g_{\mu \nu}^{(4)}(x) d x^{\mu} d x^{\nu}+R_{c}^{2} g_{i j}^{(d)}(y) d y^{i} d y^{j} .
$$

Here $R_{c}$ is the physical curvature radius of the CHM, and $g_{i j}(y)$ is therefore the metric on the CHM normalized so that its Ricci scalar is $\mathcal{R}=-1$.

Clearly, unlike Euclidean geometry, hyperbolic geometry has an intrinsic scale - the radius of curvature $R_{c}$. One therefore expects that if there is a gap in the graviton spectrum, then

$$
m_{\text {gap }}=\mathcal{O}\left(R_{c}^{-1}\right) .
$$

In $d=2$ and 3 (and probably in $d>3$ as well), there is a countable infinity of CHMs with volumes distributed approximately uniformly from a finite minimum value to infinity (in units of $R_{c}^{d}$ ). An important property of hyperbolic geometry is that at large distances volume grows exponentially with radius. As an example, note that in $H^{d}$, the volume internal to a $d-1$-sphere of radius $L \gg R_{c}$ is given by

$$
V(L) \sim R_{c}^{d} e^{\beta}
$$

where

$$
\beta \equiv\left[\frac{(d-1) L}{R_{c}}\right]
$$

The volume of a CHM is therefore of the same form (5), where $\beta$ is a constant determined by topology and related to the maximum spatial extent of the manifold. $e^{\beta}$ is a measure of the manifold's "complexity"; in $d=2$, it is proportional to the Euler characteristic of the manifold.

While primarily motivated by attractive particle physics features, this construction admits a host of interesting cosmological possibilities. In this letter we address the entropy, flatness and homogeneity problems in the context of models with compact hyperbolic extra dimensions. We argue that in this particular class of spatial manifolds, with generic particle-physics content, well-motivated initial conditions lead to observable universes that are old, flat and homogeneous, like our own. 


\section{Cosmological Problems and Extra Dimen- sions}

To understand how this picture works, let us first review why inflation is so successful. During an inflationary epoch [1] the universe expands superluminally by a large factor, meanwhile supercooling and "storing" energy in the inflaton field. After inflation, decay of the inflaton field results in the release of this stored energy into relativistic particles and an enormous increase in the total entropy of the universe. This leaves the entropy density of the universe everywhere much higher than if the universe had cooled adiabatically while undergoing a standard FRW expansion by the same factor. As a result of this expansion and entropy production, the large-scale homogeneity, flatness and entropy problems of cosmology are resolved (For a discussion see, for example, 91). It is not possible to obtain such a result from ordinary subluminal expansion, since this would require maintaining a constant entropy density through the expansion, in violation of the $3+1$ dimensional Einstein equations.

\subsection{The Entropy Problem}

One alternative attempt to solve at least some of the cosmological problems involves traditional Kaluza-Klein theories [10]-13]. The idea is that the universe possesses extra spatial dimensions beyond the three that we observe. Some of these extra dimensions may be contracting while our 3 dimensions are expanding. In this process, entropy could be squeezed out of the contracting extra dimensions, filling the three expanding ones, although it remains to understand the existence of the large total entropy in the universe.

In the model of [12] the metric is taken to be

$$
d s^{2}=-d t^{2}+a(t)^{2} d x_{\mu} d x^{\mu}+b(t)^{2} d y_{i} d y^{i}
$$

with $\mu=0, \ldots, 3, i=1, \ldots, d$. Both scale factors ( $a$ for the ordinary and $b$ for the extra space) are dynamical, and the evolution begins from zero volume, i.e. an initial singularity. The scale factor of the extra space reaches a maximum value and recollapses to a final singularity. As $b$ approaches the final singularity, a goes to infinity. This dynamics is such that the total volume,

$$
V_{\text {ТОТ }} \propto a^{3} b^{d} \equiv \sigma^{d+3},
$$


actually decreases, i.e. the extra scale factor is decreasing more rapidly than the ordinary one is increasing. (Here, $\sigma$ is the geometric mean scale factor.)

It should be mentioned that the classical equations are not to be believed all the way back to the initial time $t_{0}=0$, where quantum effects could change the whole picture. Therefore, one imagines starting from some finite time, perhaps at an energy scale close to the fundamental energy scale of $M_{F}$. Also, for a realistic theory some stabilization mechanism for the extra dimensions is required. This mechanism would prevent $b$ from becoming arbitrarily small. Since the fundamental physics is governed by the scale of $M_{F}$, it seems reasonable to expect $b$ to stabilize close to $M_{F}^{-1}$. Nevertheless, despite these considerations, a careful analysis of such cosmologies [12] shows that there is insufficient $3+1$ dimensional entropy production in these models to solve the entropy problems.

However, in the context of large extra dimensions, the very large volume of the extra dimensions is a source of much greater entropy than in traditional Kaluza-Klein theories. Also, there is a new effect. Entropy will continue to fall onto the brane even after the stabilization of the extra dimensions. The massive gravitational modes (Kaluza-Klein excitations), which are nevertheless massless from the $4+d$ point of view, cannot decay into two other massless particles if the extra-dimensional momentum is conserved. This implies that the massive gravitons can live for a very long time since they can not decay into the empty bulk. However, the presence of the brane breaks the translational invariance and allows momentum non-conservation in the extra dimensions if decay takes place on the brane. The decay of these modes would be preferentially to Standard Model particles propagating on the brane, or to these plus the graviton zero mode, which is just the ordinary 4-dimensional graviton. The coupling of gravitational modes to non-gravitational modes is typically unsuppressed compared to the coupling to other gravitational modes. Since there are many light non-gravitational modes on the brane, and only one light gravitational mode on the brane, we expect most decays of bulk gravitational modes to deposit their entropy in Standard Model fields. Finally, given that $M_{F} \geq \mathrm{TeV}$, the universe will (as shown below) thermalize before nucleosynthesis and then evolve normally after the end of the entropy condensation era. 
We will consider this phenomenon in detail, in the specific case that both the extra dimensions and our FRW space are described by CHMs. Thus, we will write their respective volumes as

$$
\begin{aligned}
& V_{\mathrm{FRW}}=a(t)^{3} e^{\alpha} \\
& V_{\text {extra }}=b(t)^{d} e^{\beta},
\end{aligned}
$$

where $a(t)$ and $b(t)$ are the respective curvature scales, and $\alpha$ and $\beta$ are topological constants. To understand this better, let us divide the evolution of the universe into several different eras. We imagine that the universe appears, in the sense that it's geometry can first be treated classically, at time $t_{0}$. The era of dynamical evolution of the ordinary and extra spaces begins at $t_{0}$ and ends at $t_{1}$ when the extra dimensions are stabilized. After stabilization, the era during which massive KK modes dominate follows from $t_{1}$ to $t_{2}$, at which point the massive KK modes decay, and the entropy moves from the bulk to the brane. This leads into the usual radiation dominated era, from $t_{2}$ to $t_{3}$, and the matter dominated era, from $t_{3}$ to $t_{4}$ (see Fig. 2.1).

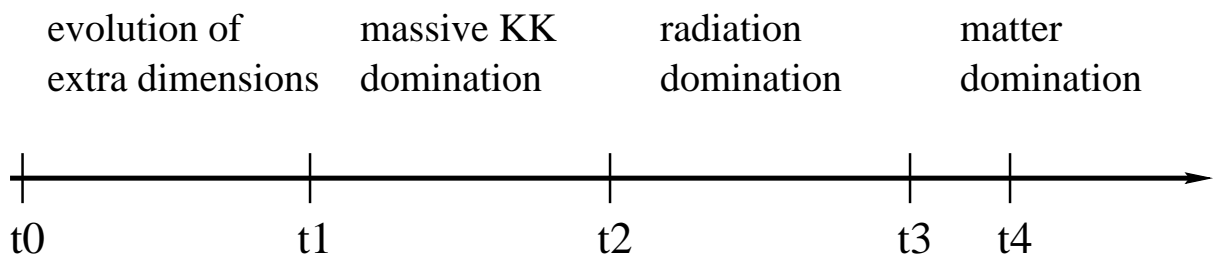

KK decay

Figure 1: Dynamical evolution of a universe with large extra dimensions

It has been argued that the maximum entropy which can be contained in a space is equal to the area of the boundary of the space in fundamental units. This is the Holographic Principle [14], which we may use to provide a bound on the total initial entropy of the universe. The Holographic Principle asserts that the total entropy grows not as the volume but as the area of the extradimensional manifold. The attractive feature of $\mathcal{M}_{\text {extra }}$ and $\mathcal{M}_{\text {FRW }}$ being of the form $H^{n} / \Gamma$ is that the area of a large CHM is approximately equal to the volume (up to geometric constants of order unity) in fundamental units. Further, the area of a CHM, like its volume, grows exponentially with radius. Thus the maximal total initial entropy is

$$
S_{0}^{\max }=g_{0} a_{0}^{3} e^{\alpha} b_{0}^{d} e^{\beta} T_{0}^{d+3}
$$


where $g_{i}(i=0,1,2)$ is the density of states for a given era. This is also a reasonable estimate for the actual value of $S_{0}$, and we will take $S_{0}=S_{0}^{\max }$. Requiring $S_{0}$ to be at least $10^{88}$ (which is the entropy within the horizon today), not taking the contribution from $g_{0}$ into account, we infer

$$
e^{\alpha+\beta} \gtrsim 10^{88}
$$

if $a_{0}^{-1} \sim b_{0}^{-1} \sim T_{0} \sim M_{F} \sim \mathrm{TeV}$. Relation (1) implies that $\beta$, a topological constant of the extra space, should not be larger than 80 . However, there is no such constraint on $\alpha$, and so the large initial entropy can be achieved within the holographic limit with $\alpha \gtrsim 130$. (Alternatively, one could drop the assumption that the FRW sub-manifold is hyperbolic, and this becomes merely a lower limit on its total volume.

This brings us to the cosmological entropy problem - the need for the enormous dimensionless number $10^{88}$ ) to specify the state of our universe [9]. We see that while we have not arrived at a dynamical solution of the problem, we have found that the problematically large dimensionless number may reasonably be regarded as the exponential of a much smaller dimensionless number - the ratio of the size of the small compact dimensions of spacetime to their curvature scale. One may of course ask why $\alpha$ and $\beta$ should be so large. Since there are a countable infinity of two- and three- dimensional CHM-s with arbitrarily large volume, a better question might perhaps be why $\alpha$ and $\beta$ are so small. Sidestepping the possible anthropic arguments, we suggest that such questions can only truly be answered in the context of a quantum cosmology incorporating dynamical topology change.

The total entropy in the universe for $t_{1}<t<t_{2}$ is

$$
S_{1}=g_{1} a_{1}^{3} b_{1}^{d} e^{\alpha+\beta} T_{1}^{3+d},
$$

where $a_{i}, b_{i}$ denote $a\left(t_{i}\right)$ and $b\left(t_{i}\right)$ respectively. Now, as $t \rightarrow t_{2}$ the universe approaches a temperature $T_{*}$, at which the massive KK modes decay. During this decay, entropy is not conserved, and so we must estimate the temperature on the brane after decay. To do this, note that energy density is conserved during the decay, and make the approximation that the decay is instantaneous, so that at $t=t_{2}$ the temperature undergoes a rapid change from $T_{*}$ to $T_{2}$, after which the universe ceases to be matter dominated (since the massive KK modes responsible for this have now decayed). Equating the energy densities at $t_{2}$, before and after the decay of the KK modes yields

$$
\rho_{*} \equiv g_{*} T_{*}^{3} M_{K K}=g_{2} T_{2}^{4} \equiv \rho_{2},
$$


where $g_{*} T_{*}^{3}$ measures the number density of KK modes at temperature $T_{*}$. Thus,

$$
T_{*}=\left(\frac{g_{2}}{g_{*}}\right)^{1 / 3}\left(\frac{T_{2}^{4}}{M_{K K}}\right)^{1 / 3} .
$$

We must now require that $T_{2} \geq 1 \mathrm{MeV}$, when the usual radiation-dominated era begins, so that the results of standard big bang nucleosynthesis are not changed.

The total entropy of the universe after the decay of KK modes is

$$
S_{2}=g_{2} \frac{M_{K K}}{T_{2}} S_{*}
$$

where $S_{*}$ is the total entropy just before the decay

$$
S_{*}=g_{*} a_{*}^{3} b_{*}^{d} e^{\alpha+\beta} T_{*}^{3+d} .
$$

Here, $a_{*}=a_{2}$ because the decay is instantaneous and $b_{*}=b_{1}$ because the extra dimensions are stabilized after $t=t_{1}$. An aspect of the entropy problem closely related to the homogeneity problem is that the entropy contained within the horizon at early times (for example recombination or nucleosynthesis) was much less than the present value of about $10^{88}$. This means that the present Hubble volume consisted of many causally disconnected regions at some earlier time and causal processes could not produce the smooth universe we see today. We must therefore require not only that the total entropy in the universe be at least $10^{88}$, but also that the entropy within the horizon, at the beginning of the ordinary evolution, i.e. $t=t_{2}$, is $10^{88}$. The horizon volume in CHM geometry, i.e. a 3-dimensional sphere of radius $L(t)$, cut out of an $H^{3}$ of curvature radius $a(t)$ is

$$
V_{L}=\pi a(t)^{3}\left[\sinh \left(\frac{2 L(t)}{a(t)}\right)-\frac{2 L(t)}{a(t)}\right]
$$

where the horizon radius $L$ is given by

$$
L(t)=a(t) \int \frac{d t}{a(t)}
$$

while $a(t)=a_{0}\left(\frac{t}{t_{0}}\right)^{p}$. The coefficient $p$ depends on dynamics and it is unknown for $t_{0}<t<t_{1}$ and $p=2 / 3$ for $t_{1}<t<t_{2}$. At the moment $t=t_{2}$, practically the whole entropy has already fallen onto the brane. Thus, if by then the 3 -horizon volume $V_{L}$ contains a curvature volume $V_{\text {FRW }}$ within 
it, the entropy problem is solved because $V_{\mathrm{FRW}}$ already contains entropy of $10^{88}$. From (9) and (17) we see that this requires $t_{2} / t_{0}$ to be greater than $10^{3}$ and $10^{4}$ for $p=1 / 2$ and $p=2 / 3$ respectively, which can be easily satisfied with any reasonable dynamics. For example, one possible choice $T_{1} \sim \mathrm{TeV}$ and $T_{2} \sim 100 \mathrm{MeV}$, which also explains the flatness and homogeneity of the universe (see subsections (2.2) and (2.3)), gives $t_{2} / t_{1} \sim 10^{6}$.

\subsection{The Flatness Problem}

Let us now turn to the flatness problem - the fact that observations today show no trace of a curvature of the universe although Einstein's equations dictate that even a small initial curvature term quickly dominates over matter or radiation density in the evolution of the universe.

Expressed in fundamental units, the initial energy density of the universe is

$$
G_{4+d} \rho_{0}=\frac{g_{0}}{M_{F}^{d+2}} T_{0}^{4+d}
$$

where $G_{4+d}$ is the fundamental (4+d dimensional) gravitational constant and $g_{0}$ is the density of states. The appropriately redshifted energy density today is

$$
G_{4+d} \rho_{4}=\frac{g_{0}}{M_{F}^{d+2}} T_{0}^{4+d}\left(\frac{\sigma_{0}}{\sigma_{1}}\right)^{d+4}\left(\frac{a_{1}}{a_{2}}\right)^{3}\left(\frac{a_{2}}{a_{3}}\right)^{4}\left(\frac{a_{3}}{a_{4}}\right)^{3},
$$

where we have used $\rho \sigma^{d+4}=$ constant for $t_{0}<t<t_{1}$ and correspondingly redshifted powers in the matter and radiation dominated eras. On the other hand, the magnitude of the appropriately redshifted curvature term today is

$$
\frac{1}{a_{4}^{2}}=\frac{1}{a_{0}^{2}}\left(\frac{a_{0}}{a_{1}}\right)^{2}\left(\frac{a_{1}}{a_{2}}\right)^{2}\left(\frac{a_{2}}{a_{3}}\right)^{2}\left(\frac{a_{3}}{a_{4}}\right)^{2} .
$$

Note that unlike inflationary models where the solutions of the entropy problem and flatness problem are closely linked, the large volume of the extra dimensions implied by our reformulation of the entropy problem does not imply a small value for the initial curvature $a_{0}^{-2}$, nor consequently the present curvature $a_{4}^{-2}$. The flatness problem therefore requires further consideration.

If we require that the evolution of the extra dimensions not disturb the usual thermal history of our universe we need

$$
\begin{aligned}
& T_{4}=10^{-3} \mathrm{eV} \\
& T_{3}=10 \mathrm{eV} \\
& T_{2} \gtrsim 1 \mathrm{MeV} .
\end{aligned}
$$


Now, if we assume that the total entropy is conserved for $t_{0}<t<t_{1}$, i.e. $S_{0}=S_{1}$, we obtain

$$
\left(\frac{\sigma_{0}}{\sigma_{1}}\right)=\left(\frac{g_{1}}{g_{0}}\right)^{1 /(3+d)}\left(\frac{T_{1}}{T_{0}}\right) .
$$

A similar consideration for $t_{1}<t<t_{*}$ yields

$$
\left(\frac{a_{2}}{a_{1}}\right)^{3}=\left(\frac{a_{*}}{a_{1}}\right)^{3}=\left(\frac{g_{1}}{g_{*}}\right)\left(\frac{T_{1}}{T_{*}}\right)^{d+3},
$$

We used $b_{2}=b_{*}=b_{1}$ where $b_{1}$, the curvature scale at late times (including currently), characterizes the low-energy mass of KK modes

$$
M_{K K} \sim b_{1}^{-1}
$$

with $\beta$ constrained by relation (11)

$$
e^{\beta}=\left(\frac{M_{P l}}{M_{F}}\right)^{2}\left(\frac{M_{K K}}{M_{F}}\right)^{d} .
$$

On the other hand, during the matter dominated era $t_{1}<t<t_{2}$, when the extra dimensions are frozen, the relationship between the scale factor and the age of the universe is:

$$
\left(\frac{a_{2}}{a_{1}}\right)=\left(\frac{t_{2}}{t_{1}}\right)^{2 / 3}
$$

with

$$
\begin{aligned}
t_{2} & \equiv \tau_{K K} \sim \frac{M_{P l}^{2}}{M_{K K}^{3}} \\
t_{1} & \sim\left(G_{4+d} \rho_{1}\right)^{-1 / 2}=\left[\frac{g_{0}}{M_{F}^{d+2}} T_{0}^{4+d}\left(\frac{\sigma_{0}}{\sigma_{1}}\right)^{d+4}\right]^{-1 / 2} .
\end{aligned}
$$

Eliminating $T_{1}$ using (24) and (27), we can express all the relevant quantities in terms of dynamical variables. Thus,

$$
\begin{gathered}
\frac{\sigma_{0}}{\sigma_{1}}=\frac{1}{g_{*}}\left(\frac{M_{K K}^{6} M_{F}^{d+2}}{T_{*}^{d+3} M_{P l}^{4} T_{0}}\right), \\
\left(\frac{a_{2}}{a_{0}}\right)^{3}=\left(\frac{g_{0}}{g_{*}}\right)\left(\frac{b_{0}}{b_{1}}\right)^{d}\left(\frac{T_{0}}{T_{*}}\right)^{d+3}, \\
\left(\frac{a_{2}}{a_{1}}\right)^{3}=\left(\frac{M_{P l}^{4}}{M_{K K}^{6}}\right) \frac{g_{0}}{M_{F}^{(d+2)}} T_{0}^{(4+d)}\left(\frac{\sigma_{0}}{\sigma_{1}}\right)^{(d+4)} .
\end{gathered}
$$


We can now calculate the ratio of the two terms relevant for the flatness problem:

$$
\frac{G_{4+d} \rho_{4}}{\left(1 / a_{4}^{2}\right)} \sim\left(\frac{M_{K K}}{T_{2}}\right)^{14 / 3+8 d / 9}\left(\frac{M_{K K}^{2} T_{3} T_{4}}{M_{P l}^{4}}\right)\left(b_{0} T_{0}\right)^{2 d / 3}\left(a_{0} T_{0}\right)^{2} \frac{g_{0}^{2 / 3} g_{*}^{2 d / 9}}{g_{2}^{2(d+3) / 9}}
$$

It is not difficult to choose generic values of parameters which yield this ratio significantly greater than one. However, by requiring a consistent dynamical evolution (for example $a_{0}<a_{1}<a_{2}$, values of $T_{0}$ and $T_{1}$ not much greater than the fundamental quantum gravity scale $M_{F}$ etc.) we considerably narrow the choice. One possible choice (neglecting the contribution from the density of states) $a_{0}^{-1} \sim M_{F} \sim T e V, M_{K K} \sim 27 M_{F}, d=7, T_{0} \sim 4 M_{F}$, $b_{0} \sim 5 \times 10^{5} M_{F}^{-1}$ and $T_{2} \sim 130 \mathrm{MeV}$ gives the numerical value of this ratio to be about 10 which reproduces the current flatness of the universe. Note that although some fine tuning of $b_{0}$ is present, the situation is much better than in the ordinary 3 -dim case where we needed to tune $a_{0}$ to about 30 orders of magnitude.

More heuristically, the explanation of cosmological flatness in this picture is the enormous injection of entropy into the brane by the combination of the collapse of the extra-dimensions to their final value, and the subsequent decay of the KK modes in the bulk into modes on the brane.

We should briefly comment on the possibility that in the context of extra dimensions the flatness problem may not be present $a b$ initio. This is because the structure of spacetime may be a solution of the highly non-linear string equations of motion with some configuration of sources (eg. D-branes). Suppose that the structure of the total space-time is

$$
\Sigma_{4+d_{1}+d_{2}}=R \times \mathcal{M}_{\mathrm{FRW}}^{3} \times \mathcal{M}_{\text {extra1 }}^{d 1} \times \mathcal{M}_{\text {extra2 }}^{d 2}
$$

where $\mathcal{M}_{\text {extra1 }}^{d 1}$ is CHM. The source configuration may then require some specific $\mathcal{M}_{\text {extra1 }}^{d 2}$, such as a hypersphere. The zero-global-curvature of $\mathcal{M}_{\text {FRW }}^{3}$ may then be merely a consistency condition of the solution, and we would then need only explain the absence of local inhomogeneities. 


\subsection{The Homogeneity Problem}

Now consider the homogeneity problem. We will see that the process of entropy injection from extra dimensions embeds a huge number of initially uncorrelated layers into the brane universe. Thus, the homogeneity of the brane universe today may be greatly enhanced over that expected from the standard cosmology.

We assume that in the formation of the universe, there exists some correlation scale $\xi \simeq M_{F}^{-1}$, on which fluctuations in all quantities (e.g. $\rho$ ) are correlated, but above which all fluctuations are independent. We assume further that the fluctuations on this scale are $\mathcal{O}(1)$. In the absence of a complete underlying theory of the formation of the universe, we offer no proof of this assumption. Other equally reasonable assumptions could undoubtedly be made.

Consider then a primordial fluctuation in homogeneity $\delta \rho_{0} / \rho_{0}$. The magnitude of this fluctuation, when evolved to the present day, is suppressed by a huge number $\sqrt{n}$, where $n$ is the number of appropriately redshifted fundamental volumes (of radii $1 / M_{F}$ ) contained in the horizon volume of the 3 -space at some late time $t_{4}$ (which we take to be the time of last scattering, when $T_{4} \sim 1 \mathrm{eV}$.)

$$
n=\frac{e^{\beta} b_{0}^{d}\left(t_{4} / t_{3}\right)^{3}\left(t_{3} / t_{2}\right)^{3}\left(t_{2} / t_{1}\right)^{3}\left(t_{1} / t_{0}\right)^{3} t_{0}^{3}}{\xi^{3+d}\left(\sigma_{1} / \sigma_{0}\right)^{d+3}\left(a_{2} / a_{1}\right)^{3}\left(a_{3} / a_{2}\right)^{3}\left(a_{4} / a_{3}\right)^{3}} .
$$

In addition, the primordial fluctuations contain a factor which grows in time. In the radiation and matter dominated eras, the growth factors are $t_{f} / t_{i n}$ and $\left(t_{f} / t_{i n}\right)^{2 / 3}$ respectively, where the subscripts "in" and "f" stand for "initial" and "final" [15]. Thus, the fluctuations at the horizon scale are:

$$
\left(\frac{\delta \rho}{\rho}\right)_{H o r\left(t_{4}\right)} \sim \frac{1}{\sqrt{n}}\left(\frac{t_{4}}{t_{3}}\right)^{2 / 3}\left(\frac{t_{3}}{t_{2}}\right)\left(\frac{t_{2}}{t_{1}}\right)^{2 / 3}\left(\frac{t_{1}}{t_{0}}\right)^{k}\left(\frac{\delta \rho}{\rho}\right)_{H o r\left(t_{0}\right)}
$$

where we have assumed that the universe was effectively matter dominated for $t_{1}<t<t_{2}$, when the radius of extra dimensions was frozen and most of the KK excitations were massive. We leave the coefficient $k$ undetermined for now. 
Using the relation between the scale factor and time for $t_{0}<t<t_{1}$ $\left(t_{1} / t_{0}\right)=\left(a_{1} / a_{0}\right)^{m}$, where the coefficient $m$ is also undetermined for now, we obtain:

$$
\begin{aligned}
\left(\frac{\delta \rho}{\rho}\right)_{H o r\left(t_{4}\right)} \sim & e^{-\beta / 2}\left(b_{0} M_{F}\right)^{-d / 2}\left(t_{0} M_{F}\right)^{-3 / 2}\left(\xi M_{F}\right)^{(3+d) / 2}\left(\frac{\sigma_{1}}{\sigma_{0}}\right)^{(d+3) / 2} \\
& \times\left(\frac{a_{4}}{a_{3}}\right)^{1 / 4}\left(\frac{a_{3}}{a_{2}}\right)^{1 / 2}\left(\frac{a_{2}}{a_{1}}\right)^{1 / 4}\left(\frac{a_{1}}{a_{0}}\right)^{m(k-3 / 2)}\left(\frac{\delta \rho}{\rho}\right)_{\operatorname{Hor}\left(t_{0}\right)}
\end{aligned}
$$

The values for the unknown coefficients $m$ and $k$, in the the ordinary 3 -dim universe are $m=3 / 2, k=2 / 3$ for the matter dominated and $m=2$, $k=1$ for the radiation dominated universe. In the presence of the extra dimensions these numbers are different but we assume that $m \geq 1$ and $k \leq 1$ in order not to violate the causality. Thus, in the most conservative case, $m=k=1$, we have:

$$
\begin{aligned}
& \left(\frac{\delta \rho}{\rho}\right)_{H o r\left(t_{4}\right)} \sim\left(\frac{T_{2}}{M_{F}}\right)^{\frac{d^{2}}{3}+\frac{17 d}{9}+\frac{19}{6}}\left(\frac{M_{F}}{M_{K K}}\right)^{\frac{d^{2}}{12}+\frac{95 d}{36}+\frac{31}{6}}\left(\frac{M_{P l}}{M_{F}}\right)^{d+2}\left(\frac{T_{0}}{M_{F}}\right)^{\frac{d+3}{3}} \times \\
& \times\left(\frac{M_{F}^{2}}{T_{3} T_{4}}\right)^{\frac{1}{4}}\left(b_{0} M_{F}\right)^{-\frac{2 d}{3}}\left(t_{0} M_{F}\right)^{-\frac{3}{2}}\left(\xi M_{F}\right)^{\frac{3+d}{2}} \frac{g_{0}^{\frac{1}{12}} g_{2}^{\frac{1}{36}\left(24+17 d+3 d^{2}\right)}}{g_{*}^{\frac{1}{36}\left(8 d+3 d^{2}\right)}}
\end{aligned}
$$

Unlike the flatness of the universe, it is much easier to explain its homogeneity without any fine tuning. For example, neglecting the contribution from the density of states, if $d=7$, then $T_{2} \sim 100 \mathrm{MeV}$ with

$$
T_{0} \sim b_{0}^{-1} \sim t_{0}^{-1} \sim \frac{M_{K K}}{10} \sim M_{F} \sim \mathrm{TeV}
$$

gives

$$
\left(\frac{\delta \rho}{\rho}\right)_{H o r\left(t_{4}\right)}=10^{-8}\left(\frac{\delta \rho}{\rho}\right)_{H o r\left(t_{0}\right)} .
$$

which reproduces the current cosmological homogeneity if $\left(\frac{\delta \rho}{\rho}\right)_{\operatorname{Hor}\left(t_{0}\right)}$ is of order one, i.e. if initially the energy density distribution in the universe was peaked around the reasonable value of $T_{0}^{4+d}$. Note that if we use the same numbers which explain flatness then we obtain an even more homogeneous universe (with the $10^{-8}$ above replaced by $10^{-40}$ ). Any initial inhomogeneities are thus smoothed out beyond detection purely by the very large statistical averaging inherent in the collapse of entropy onto the brane. 
It is worth pointing out that this relationship between the horizon and flatness problems is opposite to that in inflation. There it is the horizon problem which is more difficult to solve, and the required expansion yields an extremely flat universe. This is the reason that open universes from inflation [16] require more than one period of inflation. In our case, one could imagine an open universe resulting from the dynamics we have described, with the horizon problem still remaining easily solved, as can be seen from the absence of $\alpha$ in Eq. (37)

For comparison, a similar calculation for the ordinary FRW case, without the extra dimensions, and in which the fundamental volume has radius $M_{\mathrm{pl}}^{-1}$, gives

$$
\left(\frac{\delta \rho}{\rho}\right)_{H o r\left(t_{4}\right)} \sim \frac{M_{\mathrm{pl}}^{1 / 2}}{T_{3}^{1 / 4} T_{4}^{1 / 4}} \sim 10^{14}\left(\frac{\delta \rho}{\rho}\right)_{H o r\left(t_{0}\right)} .
$$

\section{Conclusions}

We have examined the problems of standard cosmology in a class of braneworld models in which both the ordinary 3-dimensional universe and the extra dimensions are compact hyperbolic manifolds. In this context, many of the problems of the standard cosmology are addressed in a new way. First, the evolution of the extra-dimensional space at early cosmic times can inject a huge entropy onto the Standard-Model-supporting brane, greatly enhancing the entropy inside the effective 3-dimensional horizon. This allows us to reexpress the large dimensionless number $S=10^{88}$ as an exponential of the ratio of the linear size of the extra-dimensions of spacetime to their curvature scale - a rather smaller dimensionless number. Although, unlike in inflation, we have offered no dynamical solution of the cosmological entropy problem (the existence of the large dimensionless number $10^{88}$ ), we have reformulated it in a way in which it may be less numerically daunting, and which suggests a path for an alternative solution - a dynamical understanding of topology change in the quantum creation of the universe. Interestingly, the necessary initial entropy of the full space need not exceed the holographic limit. Second, injection of the large initial entropy onto the brane from the extra dimensions results in a very homogeneous brane universe today. Finally, for reasonable parameters of the model (with a mild fine tuning in the extra space curvature scale), the curvature of the 3-manifold is small today, and so the flatness of the universe can be understood. Thus, the evolution of the extra dimensional 
space in these models can result in a low-energy universe, as seen from our brane, which is flat, full, and homogeneous. Moreover, within this framework, the recent solutions to the hierarchy problem can be readily realized. We have offered no detailed fundamental model of the dynamics of the spacetime during the period before the extra dimensions are frozen, nor have we offered any calculation of the spectrum of primordial fluctuations that would arise in such a model. We suggest that the appropriate dynamical models could and should be found, and that sources for fluctuations do exist, at least in the dynamics of the brane. This, and many other outstanding questions are the subject of ongoing and future investigations.

We would like to thank Nemanja Kaloper for numerous invaluable conversations and suggestions during the early stages of this project; Andrei Linde for comments and discussions on an earlier version of this paper, and on the entropy problem and its solution in inflation; also J. Alexander, R. Brandenberger, S. Carroll, G. Gibbons, C. Gordon, J. Ratcliffe, W. Thurston, T. Vachaspati and J. Weeks for many discussions and answers to (for us) difficult questions. The work of GDS and DS was supported by a DOE grant to the particle astrophysics group at CWRU. GDS received additional support from an NSF CAREER award. MT thanks the DOE and Syracuse University for support.

\section{References}

[1] A. H. Guth, Phys. Rev. D 23, 347 (1981); A.D. Linde, Phys. Lett. B 108, 389 (1982); A. Albrecht and P.J. Steinhardt, Phys. Rev. Lett. 48, 1220 (1982).

[2] J.J. Levin and K. Freese, Phys. Rev. D47, 4282 (1993).

[3] A. Albrecht and J. Magueijo, Phys. Rev. D59 043516 (1999).

[4] I. Antoniadis, Phys. Lett. B246, 377 (1990); J. Lykken Phys. Rev. D54, 3693 (1996).

[5] N. Arkani-Hamed, S. Dimopoulos and G. Dvali, Phys. Lett. B429, 263 (1998); I. Antoniadis, N. Arkani-Hamed, S. Dimopoulos and G. Dvali, Phys. Lett. B436, 257 (1998). 
[6] L. Randall and R. Sundrum, Phys. Rev. Lett. 83, 3370 (1999); ibid 4690 (1999); J. Lykken and L. Randall, hep-th/9908076; N. Arkani-Hamed, S. Dimopoulos, G. Dvali, N. Kaloper, hep-th/9907209, Phys. Rev. Lett. 84586 (2000).

[7] I. Antoniadis and K. Benakli, Phys. Lett. B326, 69 (1994); K. Dienes, E. Dudas, and T. Gherghetta, Phys. Lett. B436, 55 (1998), Nucl. Phys. B537, 47 (1999).

[8] N. Kaloper, J. March-Russell, G.D. Starkman and M. Trodden, Phys. Rev. Lett. 85, 928 (2000); hep-th/0002001; M. Trodden, hepth/0010032.

[9] A.D. Linde, Particle Physics and Cosmology, section 1.5, (Harwood, Chur, Switzerland, 1990).

[10] E. Alvarez and M. Belén-Gavela, Phys. Rev. Lett. 51, 931 (1983).

[11] D. Sahdev, Phys. Lett. B137, 155 (1984).

[12] E.W. Kolb, D. Lindley and D. Seckel, Phys. Rev. D30, 1205 (1984)

[13] R.B. Abbott, S. Barr and S. Ellis, Phys. Rev. D30, 720 (1984).

[14] G. 't Hooft, "Dimensional reduction in quantum gravity", grqc/9310026; L. Susskind, J. Math. Phys. 36, 6377 (1995); J. D. Bekenstein, Phys. Rev. D7, 2333 (1973). R. Easther and D. A. Lowe, Phys. Rev. Lett. 82, 4967 (1999) R. Bousso, JHEP 9907, 004 (1999); ibid 028 (1999)

[15] J. M. Bardeen, Phys. Rev. D22, 1882 (1980)

[16] M. Bucher, A. S. Goldhaber and N. Turok, Phys. Rev. D 52, 3314 (1995) hep-ph/9411206. 